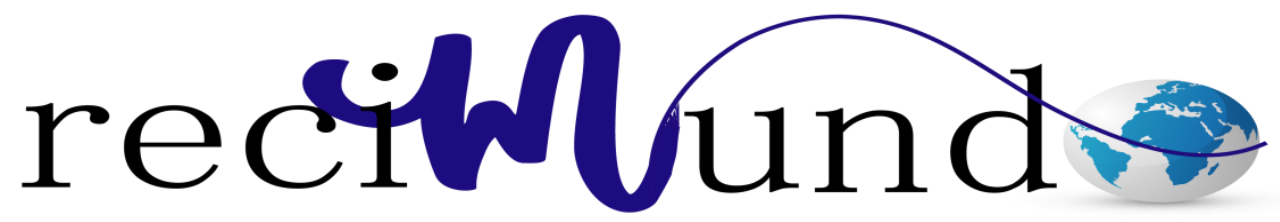

Revista Científica Mundo de la Investigación y el Conocimiento

Luis Antonio Caicedo Hinojosa a ${ }^{\text {a }}$ Janeth Reina Hurtado Astudillo ${ }^{\text {b; Rosario Del }}$ Carmen Chuquimarca Chuquimarca ${ }^{\mathrm{c}}$; Betthy Narcisa Mazacón Roca ${ }^{\mathrm{d}}$

El desarrollo de competencias profesionales basado en la gestión de medios para la realización de prácticas pre-profesionales en las carreras de la Escuela de Tecnología Medica de la facultad de Ciencias de la Salud

Revista Científica Mundo de la Investigación y el Conocimiento. Vol. 2 núm., 1, febrero, ISSN: 2588-073X, 2018, pp. 325-349

DOI: 10.26820/recimundo/2.1.2018.325-349

\author{
Editorial Saberes del Conocimiento
}

Recibido: 05/12/2017

Aceptado: 10/02/2018
a. Universidad Técnica de Babahoyo; lcaicedo@utb.edu.ec
b. Universidad Técnica de Babahoyo; jhurtado@utb.edu.ec
c. Universidad Técnica de Babahoyo; rchuquimarca@utb.edu.ec
d. Universidad Técnica de Babahoyo; bmazacon@utb.edu.ec 


\section{El desarrollo de competencias profesionales basado en la gestión de medios para la realización de prácticas pre-profesionales en las carreras de la Escuela de Tecnología Médica de la facultad de Ciencias de la Salud}

Vol. 2, núm. 1., (2018)

Luis Antonio Caicedo Hinojosa; Janeth Reina Hurtado Astudillo; Rosario Del Carmen Chuquimarca Chuquimarca; Betthy Narcisa Mazacón Roca

\section{RESUMEN}

El presente trabajo de investigación se enfoca en la ejecución de los lineamientos expuestos en el reglamento CU- SO $\mathrm{N}^{\circ} 050-\mathrm{UTB}-2015$, para la realización de prácticas pre-profesionales en las carreras de la Escuela de Tecnología Médica de la Facultad de Ciencias de la Salud de la Universidad Técnica de Babahoyo bajo un esquema de referencia en el desarrollo de competencias profesionales y la gestión de medios de procesos. Esta investigación tiene como objetivo vincular a nuestros alumnos al sector de la Salud en el Ecuador. La evaluación se desarrollara en tres fases: Evaluación por parte del Representante de la Empresa, Evaluación por parte del Supervisor delegado de la Universidad Técnica de Babahoyo y presentación de un Informe técnico de las actividades realizadas. Entre los resultados obtenidos se encuentra la redacción del convenio marco de colaboración de prácticas pre-profesionales entre la empresa y la Universidad Técnica de Babahoyo y el formato para la elaboración del informe técnico.

Palabras clave: Empresa, Prácticas, Convenio, Alumnos, Profesionales. 


\title{
EI desarrollo de competencias profesionales basado en la gestión de medios para la realización de prácticas pre-profesionales en las carreras de la Escuela de Tecnología Médica de la facultad de Ciencias de la Salud \\ Vol. 2, núm. 1., (2018) \\ Luis Antonio Caicedo Hinojosa; Janeth Reina Hurtado Astudillo; Rosario Del Carmen Chuquimarca Chuquimarca; Betthy Narcisa Mazacón Roca
}

\begin{abstract}
The present research work focuses on the execution of the guidelines set out in regulation CUSO $N^{\circ}$ 050-UTB-2015, for the realization of pre-professional practices in the careers of the School of Medical Technology of the Faculty of Sciences of the Health of the Technical University of Babahoyo under a reference scheme in the development of professional skills and the management of process media. This research aims to link our students to the Health sector in Ecuador. The evaluation will be developed in three phases: Evaluation by the Company Representative, Evaluation by the Deputy Supervisor of the Technical University of Babahoyo and presentation of a Technical Report of the activities carried out. Among the results obtained is the drafting of the framework agreement for collaboration of pre-professional practices between the company and the Technical University of Babahoyo and the format for the preparation of the technical report.
\end{abstract}

Keywords: Company, Practices, Agreement, Students, Professionals. 


\section{El desarrollo de competencias profesionales basado en la gestión de medios para la realización de prácticas pre-profesionales en las carreras de la Escuela de Tecnología Médica de la facultad de Ciencias de la Salud}

Vol. 2, núm. 1., (2018)

Luis Antonio Caicedo Hinojosa; Janeth Reina Hurtado Astudillo; Rosario Del Carmen Chuquimarca Chuquimarca; Betthy Narcisa Mazacón Roca

\section{Introducción.}

En base al Reglamento Interno de la Comisión de Vinculación con la sociedad expedido por la Universidad Técnica de Babahoyo en sesión ordinaria $\mathrm{N}^{\circ} 05$ de fecha 27 de Julio del 2015, adopto la siguiente resolución CU-SO-005-RES-050-UTB-2015 en la que se estableció aprobar el "Reglamento para realizar vinculación con la colectividad y Prácticas PreProfesionales y/o pasantías en la Universidad Técnica de Babahoyo”, presentando mediante oficio $\mathrm{N}^{\circ}$ 129-VIP-UTB-2015 de fecha 16 de Junio del 2015.

Los estudiantes de la Universidad Técnica de Babahoyo de la Facultad de Ciencias de la Salud, durante los semestres se les presenta como requisito fundamental la realización de las prácticas Pre-Profesionales en los diferentes campos de estudio relacionado con la Salud del País, por lo cual se muestra especial interés en desarrollar un esquema basado en los lineamientos del reglamento CU- SO N050-UTB-2015, para la realización de prácticas preprofesionales y en la optimización de gestión de medios, desarrollando técnicas explícitas para generar un proceso de aprendizaje basados en la cognición y meta-cognición, dando como consecuencia el desarrollo de las competencias profesionales.

Cabe mencionar que la Universidad Técnica de Babahoyo presenta dentro de sus indicadores académicos el número de convenios firmados con las empresas para la realización de las Prácticas Pre- Profesionales, con la firma de dichos convenios los estudiantes pueden realizar las diferentes prácticas que demanda cada una de sus carreras, también pueden optar por las 


\section{EI desarrollo de competencias profesionales basado en la gestión de medios para la realización de prácticas pre-profesionales en las carreras de la Escuela de Tecnología Médica de la facultad de Ciencias de la Salud}

Vol. 2, núm. 1., (2018)

Luis Antonio Caicedo Hinojosa; Janeth Reina Hurtado Astudillo; Rosario Del Carmen Chuquimarca Chuquimarca; Betthy Narcisa Mazacón Roca

cartas de intención dependiendo el caso que lo amerite. (Velásquez \& Huatuco, 2011) (EstévezArias, Medina-Chicaiza, \& González-Hernández, 2016)

Se considera de vital importancia la inclusión de las empresas e instituciones en el campo académico, dado que la educación es un proceso bidireccional en el proceso de aprendizaje, por lo cual se necesita que un número mayor de empresas e instituciones se acojan a la modalidad de convenios con la Universidad Técnica de Babahoyo, generando de esta manera un proceso de responsabilidad social con las comunidades, asociaciones, gremios y población en general, en el que los estudiantes están directamente inmersos y ávidos de conocimientos, pero se necesita que dichos actores privados y públicos permitan complementar su proceso de aprendizaje y realicen un desarrollo cíclico profesional en la difusión de los conocimientos, con lo cual se contribuirá a mejorar la praxis en salud del país, dado como consecuencia una masa crítica de profesionales incluidos en los problemas y tensiones de cada una de las áreas de dicha rama.

La realización de las Prácticas Pre-Profesionales es de importante relevancia para los procesos de aprendizajes académicos con el objetivo de afianzar los conocimientos teóricos y prácticos de los estudiantes, para que de esta manera se vinculen a su futuro campo profesional, en las diferentes actividades que su profesión lo amerita. (Ceroni Galloso , 2007) (Oliver Ventura, Santana Amargó, Ferrer Chinea, \& Ríos Obregón, 2015)

\section{Materiales y métodos.}

La evaluación se la realizará en tres fases tal como se la detalla a continuación: 


\section{El desarrollo de competencias profesionales basado en la gestión de medios para la realización de prácticas pre-profesionales en las carreras de la Escuela de Tecnología Médica de la facultad de Ciencias de la Salud}

Vol. 2, núm. 1., (2018)

Luis Antonio Caicedo Hinojosa; Janeth Reina Hurtado Astudillo; Rosario Del Carmen Chuquimarca Chuquimarca; Betthy Narcisa Mazacón Roca
A. Evaluación por parte del Representante de la Empresa. (5 puntos).

B. Evaluación por parte del Supervisor delegado de la Universidad Técnica de Babahoyo. (5 puntos).

C. Presentación de un Informe técnico de las actividades realizadas. (5 puntos)

\section{Metodología de la realización de la práctica.}

- Socialización de Prácticas Pre-Profesionales con las empresas para la obtención de convenios o cartas de intención, por parte del coordinador de las prácticas pre-profesionales.

- Visita a las empresas para revisión de propuesta de convenios o cartas de intención, por parte del coordinador de las prácticas pre-profesionales.

- Socialización de con los estudiantes sobre las prácticas Pre-Profesionales, por parte del coordinador de las prácticas pre-profesionales, coordinador de la carrera, y supervisores de la práctica.

- Gestión individual con los estudiantes para la documentación de las prácticas PreProfesionales por parte del coordinador de la práctica pre-profesional de la facultad.

- Supervisión a los estudiantes en la empresa que realiza la práctica Pre-Profesional por parte del supervisor de la universidad.

- Supervisión en la evaluación del estudiante por parte del representante de la empresa por parte del supervisor de la empresa. 


\section{EI desarrollo de competencias profesionales basado en la gestión de medios para la realización de prácticas pre-profesionales en las carreras de la Escuela de Tecnología Médica de la facultad de Ciencias de la Salud}

Vol. 2, núm. 1., (2018)

Luis Antonio Caicedo Hinojosa; Janeth Reina Hurtado Astudillo; Rosario Del Carmen Chuquimarca Chuquimarca; Betthy Narcisa Mazacón Roca

- Evaluación del supervisor delegado por la Universidad Técnica de Babahoyo al estudiante que realiza la Práctica Pre-Profesional e informe técnico emitido por el estudiante.

- Entrega de documentación por parte del estudiante de todo el proceso de prácticas preprofesional al coordinador de prácticas pre-profesional.

Con lo expuesto anteriormente los estudiantes cumplirían el requisito de las Prácticas PreProfesionales en las diferentes carreras de la Escuela de Tecnología Médica una vez terminada la actividad en la empresa, los estudiantes tendrán dos semanas para finalizar el informe técnico para lo cual podrán agruparse entre máximo 3 estudiantes por empresa. 
El desarrollo de competencias profesionales basado en la gestión de medios para la realización de prácticas pre-profesionales en las carreras de la Escuela de Tecnología Médica de la facultad de Ciencias de la Salud

Vol. 2, núm. 1., (2018)

Luis Antonio Caicedo Hinojosa; Janeth Reina Hurtado Astudillo; Rosario Del Carmen Chuquimarca Chuquimarca; Betthy Narcisa Mazacón Roca

\section{Resultados.}

Árbol de Problemas

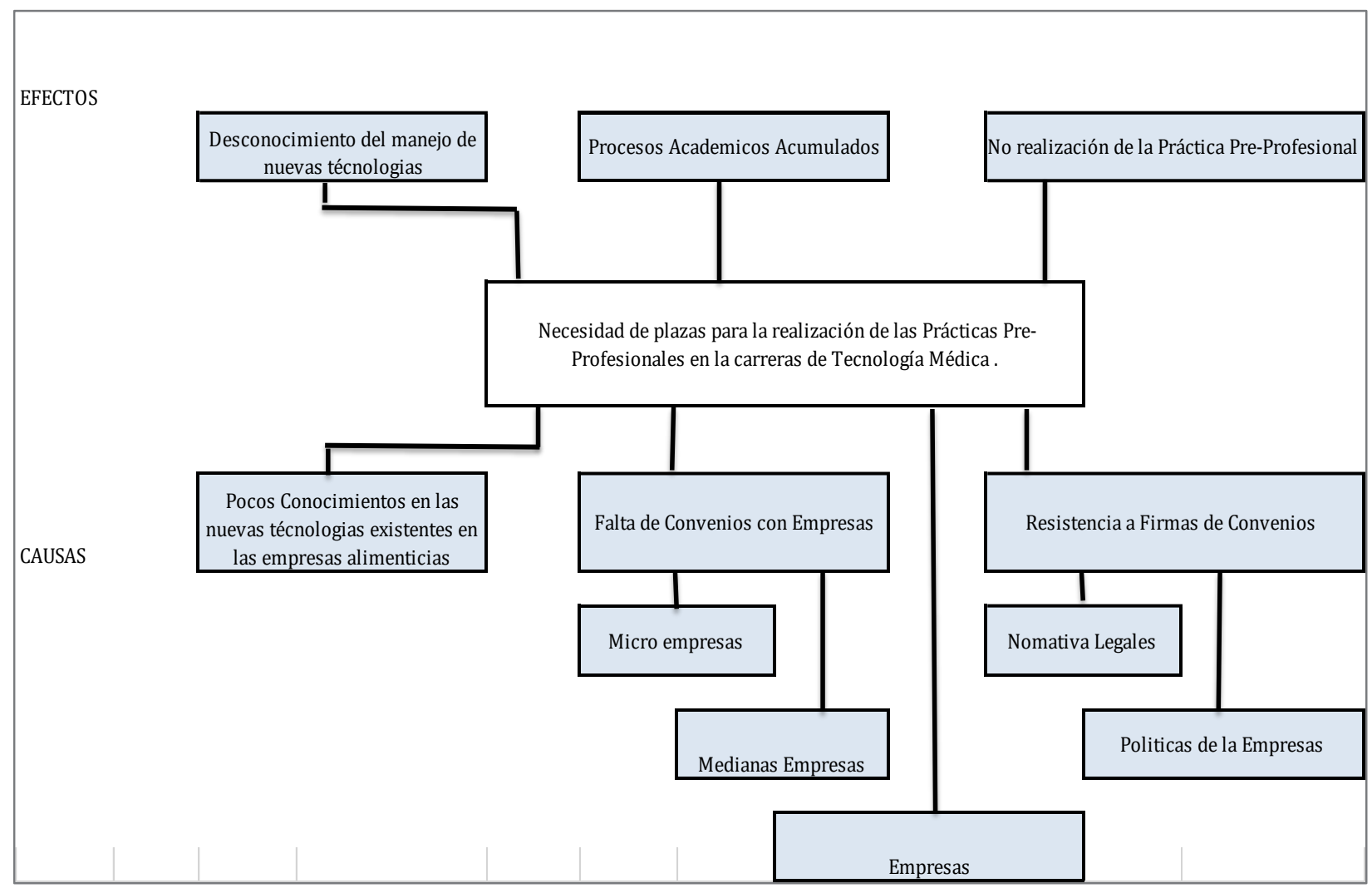

Fuente: Elaboración Propia.

\section{Árbol de Objetivos.}


El desarrollo de competencias profesionales basado en la gestión de medios para la realización de prácticas pre-profesionales en las carreras de la Escuela de Tecnología Médica de la facultad de Ciencias de la Salud

Vol. 2, núm. 1., (2018)

Luis Antonio Caicedo Hinojosa; Janeth Reina Hurtado Astudillo; Rosario Del Carmen

Chuquimarca Chuquimarca; Betthy Narcisa Mazacón Roca

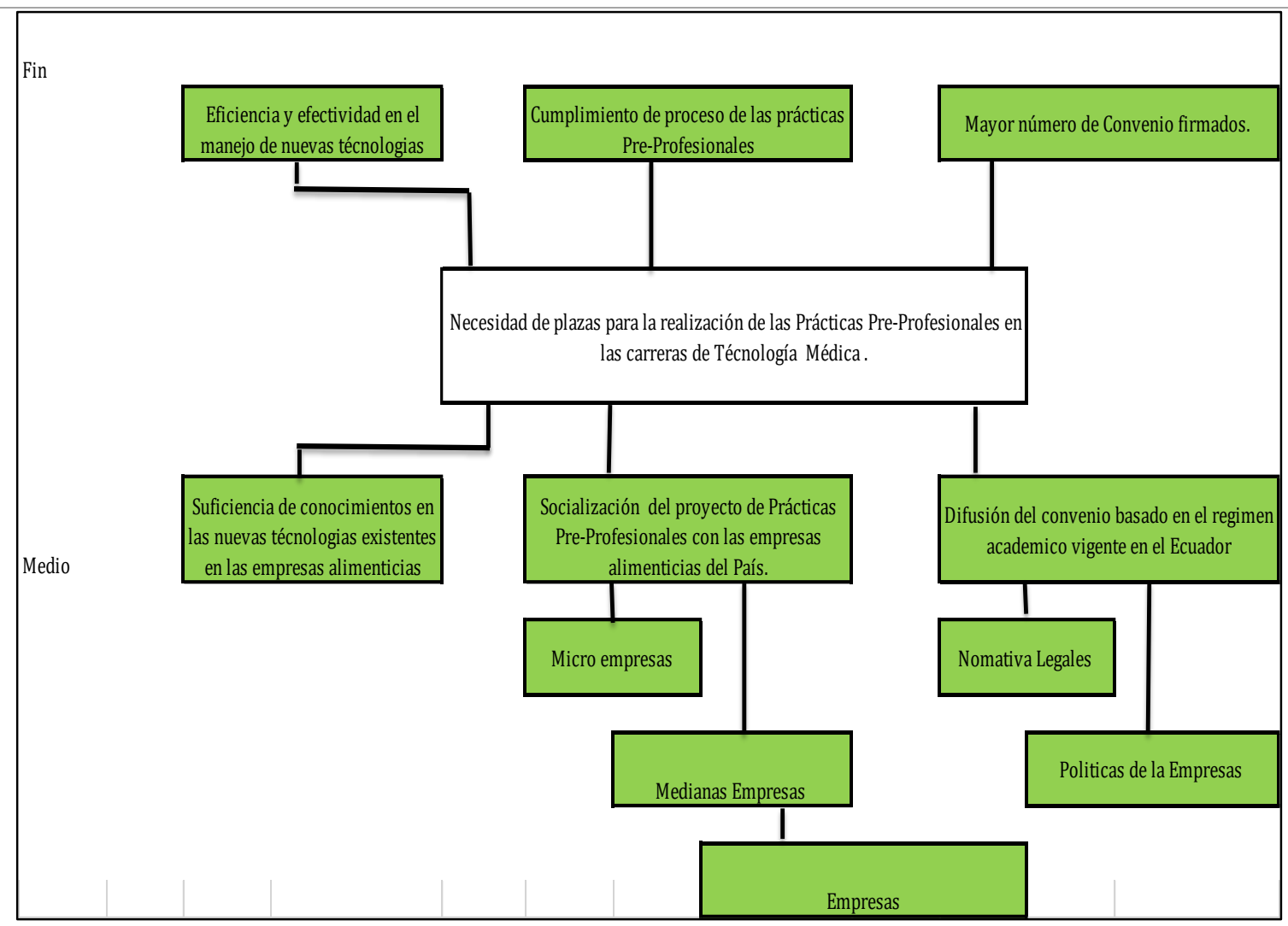

Fuente: Elaboración Propia. 
EI desarrollo de competencias profesionales basado en la gestión de medios para la realización de prácticas pre-profesionales en las carreras de la Escuela de Tecnología Médica de la facultad de Ciencias de la Salud

Vol. 2, núm. 1., (2018)

Luis Antonio Caicedo Hinojosa; Janeth Reina Hurtado Astudillo; Rosario Del Carmen Chuquimarca Chuquimarca; Betthy Narcisa Mazacón Roca

Matriz de Marco Lógico.

\begin{tabular}{|c|c|c|c|}
\hline $\begin{array}{c}\text { Resumen } \\
\text { Narrativo de } \\
\text { objetivos }\end{array}$ & Indicadores & $\begin{array}{c}\text { Medios de } \\
\text { Verificación }\end{array}$ & Supuestos \\
\hline $\begin{array}{lrr}\text { Fin } & & \\
\text { Gestionar } & & \text { el } \\
\text { número } & & \text { de } \\
\text { estudiantes en } & \text { la } \\
\text { realización } & & \text { de } \\
\text { Prácticas } & \text { Pre- } \\
\text { Profesionales. } & \end{array}$ & $\begin{array}{l}\text { Disminuir en un } \\
100 \% \text { del } \\
\text { número de } \\
\text { estudiantes en la } \\
\text { realización de } \\
\text { Prácticas Pre- } \\
\text { Profesionales }\end{array}$ & 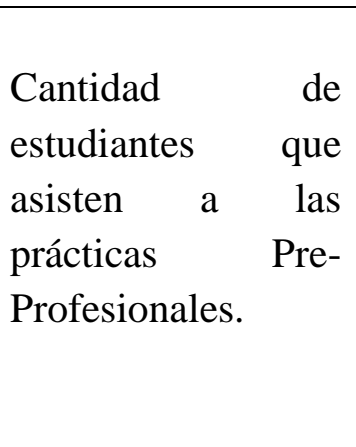 & $\begin{array}{l}\text { Que } \\
\text { comportamiento de } \\
\text { los estudiantes sea el } \\
\text { adecuado en } \\
\text { realización de la } \\
\text { Prácticas } \\
\text { Profesionales }\end{array}$ \\
\hline $\begin{array}{l}\text { Propósito } \\
\text { Aplicar los } \\
\text { conocimientos } \\
\text { teóricos y prácticos } \\
\text { recibidos en las } \\
\text { asignaturas } \\
\text { expuestas en la } \\
\text { malla curricular de } \\
\text { cada una de las } \\
\text { carreras. }\end{array}$ & 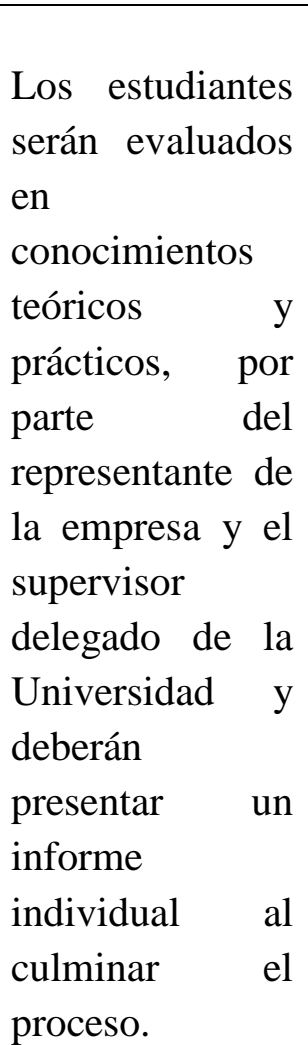 & $\begin{array}{l}\text { Informes técnico } \\
\text { por parte del } \\
\text { representante de la } \\
\text { empresa y } \text { el } \\
\text { supervisor } \\
\text { delegado de la } \\
\text { carrera. } \\
\text { Informe técnico } \\
\text { emitido por el } \\
\text { estudiante. }\end{array}$ & $\begin{array}{l}\text { Que los estudiantes se } \\
\text { sientan } \\
\text { comprometidos en el } \\
\text { desarrollo de las } \\
\text { actividades de las } \\
\text { Prácticas } \\
\text { Profesionales. }\end{array}$ \\
\hline $\begin{array}{l}\text { Componente } \\
\text { Formato }\end{array}$ & $\begin{array}{l}\text { La evaluación } \\
\text { por parte de la }\end{array}$ & $\begin{array}{lll}\text { Formato } & & \text { de } \\
\text { evaluación } & \text { de } & \text { la }\end{array}$ & $\begin{array}{lll}\text { El } \quad 10 \% & \text { de } & \text { los } \\
\text { alumnos } & \text { no } & \text { sean }\end{array}$ \\
\hline
\end{tabular}


EI desarrollo de competencias profesionales basado en la gestión de medios para la realización de prácticas pre-profesionales en las carreras de la Escuela de Tecnología Médica de la facultad de Ciencias de la Salud

Vol. 2, núm. 1., (2018)

Luis Antonio Caicedo Hinojosa; Janeth Reina Hurtado Astudillo; Rosario Del Carmen Chuquimarca Chuquimarca; Betthy Narcisa Mazacón Roca

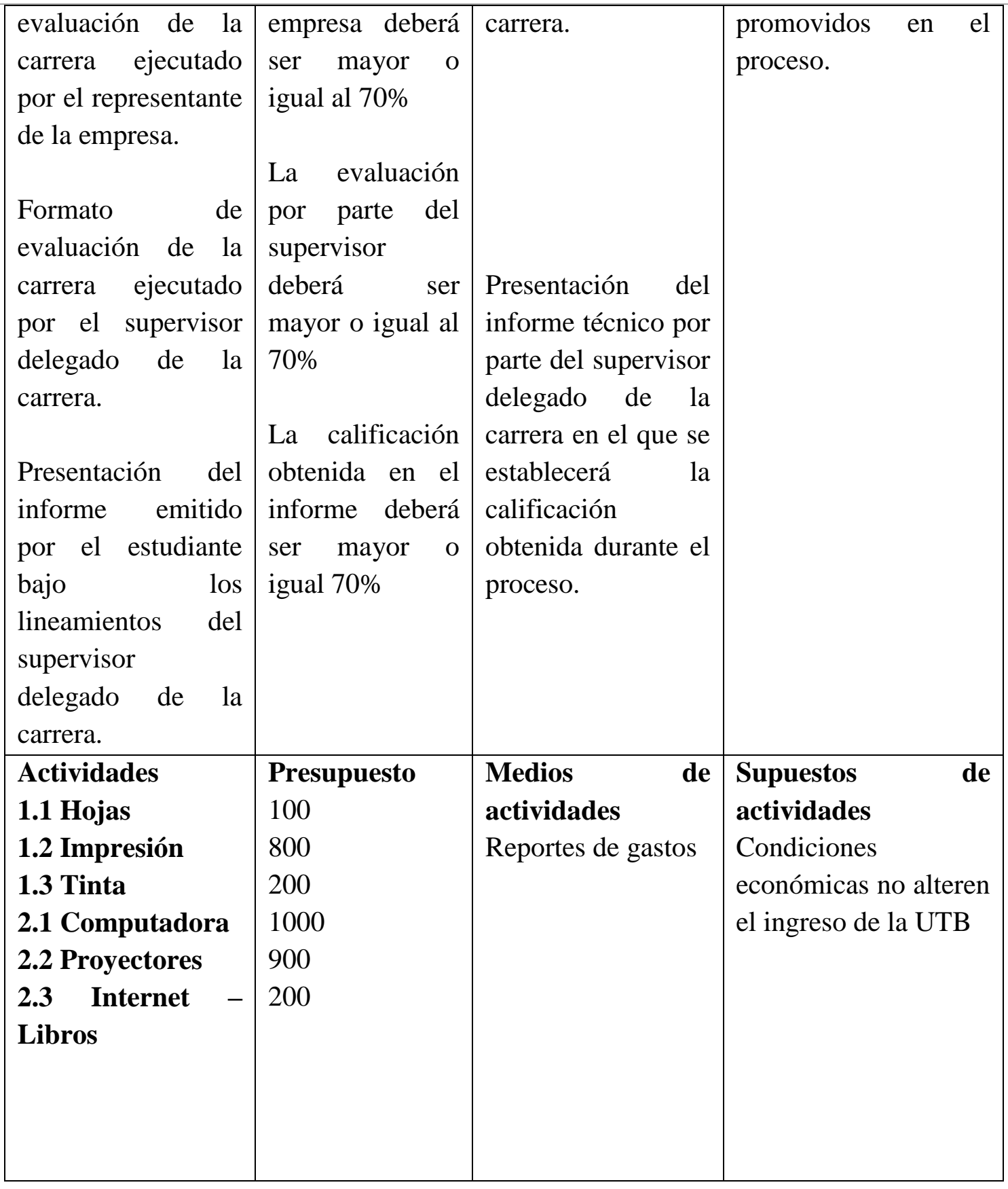

Fuente: Elaboración Propia. 
EI desarrollo de competencias profesionales basado en la gestión de medios para la realización de prácticas pre-profesionales en las carreras de la Escuela de Tecnología Médica de la facultad de Ciencias de la Salud

Vol. 2, núm. 1., (2018)

Luis Antonio Caicedo Hinojosa; Janeth Reina Hurtado Astudillo; Rosario Del Carmen Chuquimarca Chuquimarca; Betthy Narcisa Mazacón Roca

Descripción de gestión del proceso de prácticas Pre-Profesionales.

\begin{tabular}{|c|c|c|c|c|c|}
\hline No. & Actividad & $\begin{array}{c}\text { Descripción } \\
\text { Funciones }\end{array}$ & Responsable & Registro & Resultados \\
\hline 1 & $\begin{array}{l}\text { Practicas Pre } \\
\text { Profesionales } \\
\text { de } \\
\text { Coordinación }\end{array}$ & 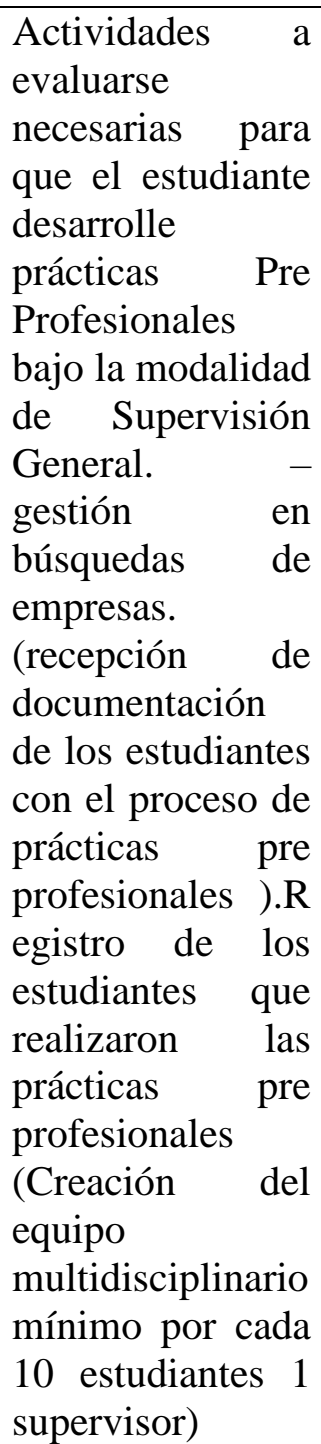 & $\begin{array}{l}\text { Docentes } \\
\text { designados (tutor } \\
\text { de la Institución } \\
\text { de Prácticas Pre- } \\
\text { Profesionales de } \\
\text { los estudiantes) }\end{array}$ & $\begin{array}{l}\text { Registro del } \\
\text { estudiante: } \\
\text { Académico.- } \\
\text { cumplimiento y } \\
\text { aprobación para } \\
\text { realizar prácticas } \\
\text { pre-profesionales }\end{array}$ & $\begin{array}{l}\text { Entrega } \\
\text { certifica cados } \\
\text { de } \\
\text { cumplimiento } \\
\text { de prácticas } \\
\text { pre } \\
\text { profesionales }\end{array}$ \\
\hline
\end{tabular}




\section{EI desarrollo de competencias profesionales basado en la gestión de medios para la realización de prácticas pre-profesionales en las carreras de la Escuela de Tecnología Médica de la facultad de Ciencias de la Salud}

Vol. 2, núm. 1., (2018)

Luis Antonio Caicedo Hinojosa; Janeth Reina Hurtado Astudillo; Rosario Del Carmen

Chuquimarca Chuquimarca; Betthy Narcisa Mazacón Roca

\begin{tabular}{|c|c|c|c|c|c|}
\hline 2 & $\begin{array}{l}\text { Practicas Pre } \\
\text { Profesionales } \\
\text { con Supervisión } \\
\text { Docente }\end{array}$ & $\begin{array}{l}\text { Actividades } \\
\text { necesarias para } \\
\text { que el estudiante } \\
\text { desarrolle } \\
\text { prácticas Pre } \\
\text { Profesionales } \\
\text { bajo la modalidad } \\
\text { con Supervisión } \\
\text { Docente. (entrega } \\
\text { de los formatos } \\
\text { de evaluación al } \\
\text { estudiante) }\end{array}$ & $\begin{array}{l}\text { Docente } \\
\text { Designado } \\
\text { (Tutor de la } \\
\text { Institución de } \\
\text { Prácticas Pre- } \\
\text { Profesionales de } \\
\text { los estudiantes). }\end{array}$ & $\begin{array}{l}\text { Registro del } \\
\text { estudiante: } \\
\text { Académico.- } \\
\text { cumplimiento y } \\
\text { aprobación para } \\
\text { realizar prácticas } \\
\text { pre- } \\
\text { profesionales. }\end{array}$ & $\begin{array}{l}\text { Oficio de } \\
\text { terminación } \\
\text { de prácticas } \\
\text { pre } \\
\text { profesionales } \\
\text { estipulando la } \\
\text { nota de } \\
\text { finalización }\end{array}$ \\
\hline 3 & $\begin{array}{l}\text { Practicas Pre } \\
\text { Profesionales } \\
\text { con } \\
\text { supervisión de } \\
\text { la empresa. }\end{array}$ & $\begin{array}{l}\text { Sub proceso en el } \\
\text { cual se definen } \\
\text { las actividades } \\
\text { para que los } \\
\text { estudiantes de la } \\
\text { Carreras escuelas } \\
\text { de realicen sus } \\
\text { prácticas Pre } \\
\text { Profesionales. } \\
\text { Supervisión en } \\
\text { empresa }\end{array}$ & $\begin{array}{l}\text { Responsable } \\
\text { técnico de la } \\
\text { empresa } \\
\text { Estudiantiles }\end{array}$ & $\begin{array}{l}\text { Registro del } \\
\text { estudiante: } \\
\text { Académico.- } \\
\text { cumplimiento y } \\
\text { aprobación para } \\
\text { realizar prácticas } \\
\text { pre-profesionales }\end{array}$ & $\begin{array}{l}\text { Entrega de } \\
\text { certificación } \\
\text { de } \\
\text { terminación } \\
\text { de prácticas } \\
\text { pre } \\
\text { profesionales } \\
\text { estipulando la } \\
\text { nota de } \\
\text { finalización, } \\
\text { número de } \\
\text { horas, fechas., } \\
\text { departamento, } \\
\text { responsable } \\
\text { técnico. }\end{array}$ \\
\hline
\end{tabular}

Fuente: Elaboración Propia. 


\section{El desarrollo de competencias profesionales basado en la gestión de medios para la realización de prácticas pre-profesionales en las carreras de la Escuela de Tecnología Médica de la facultad de Ciencias de la Salud}

Vol. 2, núm. 1., (2018)

Luis Antonio Caicedo Hinojosa; Janeth Reina Hurtado Astudillo; Rosario Del Carmen Chuquimarca Chuquimarca; Betthy Narcisa Mazacón Roca

\section{Conclusiones.}

\section{Modelo de convenio para prácticas Pre-Profesionales}

\section{CONVENIO MARCO DE COLABORACION DE PRACTICAS PRE-PROFESIONALES ENTRE LA EMPRESA XXX Y LA UNIVERSIDAD TÉCNICA DE BABAHOYO}

Intervienen a la suscripción del presente convenio, por una parte, XXX, representada por XXX, en su calidad de XXX parte a la que en adelante se le podrá denominar como "LA EMPRESA; y, por otra la Universidad Técnica de Babahoyo, representada legalmente por el Dr. Rafael Falconí Montalván, Msc; en su calidad de Rector, a quien en adelante se le podrá denominar "LA UNIVERSIDAD", respectivamente. Los intervinientes por los derechos que representan, libre y voluntariamente convienen en suscribir el presente convenio, de acuerdo a las siguientes cláusulas:

1.1. La empresa es una entidad (técnica y autónoma), cuya actividad económica es la de agroindustria. Y tiene como objetivo general desarrollar estrategias y en facilitar los medios para que el personal tanto de planta industrial y agrícola, asuman un mayor compromiso en la implementación de una cultura de gestión de la calidad y mejora continua teniendo como misión trabajar con entusiasmo para producir, con la más alta calidad, los mejores productos para nuestros consumidores; creemos en nuestro país y en nuestra cultura, nuestra mayor prioridad es el respeto a la naturaleza y el desarrollo humano. Nos empeñamos siempre en ser fuente de riqueza para nuestros clientes, proveedores y accionistas, y visión convertirnos en un referente de Ingenio azucarero en productividad y eficiencia 


\section{EI desarrollo de competencias profesionales basado en la gestión de medios para la realización de prácticas pre-profesionales en las carreras de la Escuela de Tecnología Médica de la facultad de Ciencias de la Salud}

Vol. 2, núm. 1., (2018)

Luis Antonio Caicedo Hinojosa; Janeth Reina Hurtado Astudillo; Rosario Del Carmen Chuquimarca Chuquimarca; Betthy Narcisa Mazacón Roca

1.2. La Universidad Técnica de Babahoyo, es un centro de estudios superiores, con domicilio en la ciudad de Babahoyo, Provincia de Los Ríos, creada el 05 de octubre de 1971, por Decreto Ejecutivo $\mathrm{N}^{\mathrm{o}} 1508$, publicada en el Registro Oficial $\mathrm{N}^{\circ} 327$. Se rige por la Constitución de la República, por la Ley Orgánica de Educación Superior, por su Estatuto Orgánico y los reglamentos expedidos por su organismo de cogobierno así como las Resoluciones de su máxima autoridad. La Universidad Técnica de Babahoyo tiene como misión primordial la investigación científica, además la formación profesional y técnica, la creación, difusión y desarrollo de la cultura nacional, para responder a las demandas de la sociedad ecuatoriana y mundial.

1.3. El artículo 87 de la Ley Orgánica de Educación Superior dispone: "Como requisito previo a la obtención del título, los y las estudiantes deberán acreditar servicios a la comunidad mediante prácticas o pasantías pre profesionales, debidamente monitoreadas, en los campos de su especialidad, de conformidad con los lineamientos generales definidos por el Consejo de Educación Superior. Dichas actividades se realizarán en coordinación con organizaciones comunitarias, empresas e instituciones públicas y privadas relacionadas con la respectiva especialidad".

1.4. El artículo 160 del Capítulo I del Título IX de la Ley Orgánica de Educación Superior dispone: "Corresponde a las universidades y escuelas politécnicas producir propuestas y planteamientos para buscar la solución de los problemas del país; propiciar el diálogo entre las culturas nacionales y de éstas con la cultura universal; la difusión y el fortalecimiento de sus valores en la sociedad ecuatoriana; la formación profesional, técnica y científica de sus 


\section{El desarrollo de competencias profesionales basado en la gestión de medios para la realización de prácticas pre-profesionales en las carreras de la Escuela de Tecnología Médica de la facultad de Ciencias de la Salud}

Vol. 2, núm. 1., (2018)

Luis Antonio Caicedo Hinojosa; Janeth Reina Hurtado Astudillo; Rosario Del Carmen Chuquimarca Chuquimarca; Betthy Narcisa Mazacón Roca

estudiantes, profesores o profesoras e investigadores o investigadoras, contribuyendo al logro de una sociedad más justa, equitativa y solidaria, en colaboración con los organismos del Estado y la sociedad."

1.5. La Universidad y la Empresa han convenido que los estudiantes, realicen prácticas pre profesionales en los diferentes Departamentos que se encuentre en relación con su carrera, bajo la modalidad de prácticas pre-profesionales, de acuerdo con su especialidad, de conformidad con lo que dispone el artículo 87 de la Ley de Orgánica de Educación Superior, la cual servirá de base para el fortalecimiento de sus conocimientos y complemento a su formación profesional.

\section{SEGUNDA: OBJETO.-}

El presente convenio tiene por objeto implementar un sistema de pasantías dentro de los términos establecidos en la Norma Técnica No. MRL-2012-0191 publicada en el Registro Oficial No. 841 de 29 de noviembre de 2012, relacionadas con la educación y formación, de acuerdo con la especialización que reciben, bajo la organización y control de la facultad a la que pertenece el alumno, durante un lapso determinado.

El número de pasantes requeridos será fijado por la EMPRESA, mismos que no podrán exceder de tres estudiantes.

\section{TERCERA: DESARROLLO DE LAS PASANTÍAS.-}

El horario de prácticas pre-profesionales será de máximo 8 horas diarias de lunes a viernes durante los cinco días a la semana, completando como mínimo las horas requeridas por la 


\section{EI desarrollo de competencias profesionales basado en la gestión de medios para la realización de prácticas pre-profesionales en las carreras de la Escuela de Tecnología Médica de la facultad de Ciencias de la Salud}

Vol. 2, núm. 1., (2018)

Luis Antonio Caicedo Hinojosa; Janeth Reina Hurtado Astudillo; Rosario Del Carmen Chuquimarca Chuquimarca; Betthy Narcisa Mazacón Roca

Universidad, al finalizar la Práctica Pre-Profesional. El pasante estará sujeto al horario que fijen conjuntamente las partes de conformidad con el pensum de estudios, comprometiéndose "LA EMPRESA" en emitir un cronograma en el cual se estipula la Fecha, hora, actividad que va a realizar el estudiante, departamento en el que se encuentra realizando la actividad, y responsable técnico por parte de la empresa que evaluará al estudiante.

La empresa admitirá a sus programas de Prácticas Pre-Profesionales únicamente a estudiantes que se encuentren matriculado a partir de sexto semestre de la carrera de Nutrición y Dietética de la Facultad de Ciencias de la Salud. Las pasantías se efectuarán en las dependencias de la EMPRESA.

CUARTA.- PLAZO.-

El presente Convenio tendrá una duración de 5 años, contado a partir de la fecha de su suscripción, pudiendo ser renovado por las partes por idénticos períodos, si así lo manifiestan por escrito y con por lo menos treinta días de anticipación a la fecha de su terminación, para lo cual, necesariamente se suscribirá un nuevo convenio.

QUINTA: OBLIGACIONES DE LAS PARTES.-

De la EMPRESA:

a) Brindará las facilidades a los estudiantes a fin de que puedan cumplir con sus jornadas y obligaciones universitarias, así como proveerá los insumos y materiales necesarios para la ejecución de las actividades de la Práctica Pre-Profesional. 


\section{El desarrollo de competencias profesionales basado en la gestión de medios para la realización de prácticas pre-profesionales en las carreras de la Escuela de Tecnología Médica de la facultad de Ciencias de la Salud}

Vol. 2, núm. 1., (2018)

Luis Antonio Caicedo Hinojosa; Janeth Reina Hurtado Astudillo; Rosario Del Carmen Chuquimarca Chuquimarca; Betthy Narcisa Mazacón Roca

b) Entregará al pasante un certificado sobre la aceptación y terminación de la Práctica PreProfesional del ejercicio de su pasantía, en el que se establecerán los siguientes campos, Nombre de la empresa, Nombre del Estudiante, Carrera, Facultad, Fecha de Inicio de la Práctica PreProfesional, Fecha de Terminación de la Práctica Pre-Profesional, duración en horas de la práctica, Nombre del representante de la empresa que tutorío al estudiante, Firma y Sello de la Empresa.

c) En el caso del reconocimiento económico por la empresa al estudiante es decisión de la empresa.

De la Universidad:

a) Seleccionará a los estudiantes que, cumpliendo con los requerimientos mínimos establecidos por la Empresa, realizarán las Prácticas Pre-Profesionales. Para el efecto, y aplicando el artículo 6 de la Norma Técnica que establece las directrices para la celebración de los convenios de pasantías y prácticas pre profesionales en el Sector Público, la Universidad seleccionará preferentemente a estudiantes con discapacidad, para colaborar con las actividades propias objeto de este convenio.

b) Notificará a la Empresa si uno de los estudiantes que realizan las Prácticas Pre-Profesionales abandona sus estudios o excede el número de faltas permitidas por la Universidad.

De los Pasantes: 


\section{EI desarrollo de competencias profesionales basado en la gestión de medios para la realización de prácticas pre-profesionales en las carreras de la Escuela de Tecnología Médica de la facultad de Ciencias de la Salud}

Vol. 2, núm. 1., (2018)

Luis Antonio Caicedo Hinojosa; Janeth Reina Hurtado Astudillo; Rosario Del Carmen Chuquimarca Chuquimarca; Betthy Narcisa Mazacón Roca

Además de los deberes establecidos en el artículo 21 de la Norma Técnica que establece las directrices para la celebración de los convenios de pasantías y prácticas pre- profesionales en el Sector Público, los pasantes deberán guardar confidencialidad de la información a la que pudiera tener acceso durante su permanencia en la Institución. Si el pasante incumpliere este deber, concluirá la Práctica Pre-Profesional, sin perjuicio de las demás sanciones administrativas, civiles o penales a que hubiere lugar.

\section{SEXTA: NATURALEZA DE LA PASANTÍA.-}

Conforme lo previsto en el artículo 5 de la Norma Técnica que establece las directrices para la celebración de los convenios de pasantías y prácticas pre profesionales en el Sector Público, la realización de la pasantía no crea ninguna relación laboral ni genera derechos u obligaciones laborales o administrativas entre las partes; en consecuencia, no crea ningún tipo de estabilidad laboral.

Los pasantes no perderán en ningún momento su condición de alumno.

\section{SÉPTIMA: DETALLE DE ACTIVIDADES.-}

El pasante realizará su Práctica Pre-Profesional bajo la supervisión del Jefe o Coordinador de la unidad de que se trate, realizando cualquiera de las siguientes actividades:

- Brindar apoyo y asistencia en los procesos de control, servicios, soporte o asesoría que le fueran asignados. 


\section{El desarrollo de competencias profesionales basado en la gestión de medios para la realización de prácticas pre-profesionales en las carreras de la Escuela de Tecnología Médica de la facultad de Ciencias de la Salud}

Vol. 2, núm. 1., (2018)

Luis Antonio Caicedo Hinojosa; Janeth Reina Hurtado Astudillo; Rosario Del Carmen Chuquimarca Chuquimarca; Betthy Narcisa Mazacón Roca

- Temas relacionados con la carrera de Nutrición y Dietética especialmente que se relaciones con los cuatro componentes como son:

1) Seguridad Alimentaria.

2) Desarrollo de métodos analíticos basados en técnicas instrumentales en el campo de agroalimentario.

3) Procesos Industriales Alimentarios.

4) Nutrición y salud.

En el cronograma de actividades emitido por la empresa se especificarán las actividades que cada pasante deberá realizar basado en los cuatro componentes.

OCTAVA: COORDINACIÓN Y SEGUIMIENTO -

Ambas partes designarán a un supervisor que será responsable de asegurar el debido cumplimiento de los deberes acordados en el presente instrumento.

Delegado de la Empresa:

Nombre: Olga Camposano Romero

Cargo: Gerente de Talento Humano

Dirección: Urdesa Central, Calle 6ta.\# 309 y Av. Las Lomas. 


\section{EI desarrollo de competencias profesionales basado en la gestión de medios para la realización de prácticas pre-profesionales en las carreras de la Escuela de Tecnología Médica de la facultad de Ciencias de la Salud \\ Vol. 2, núm. 1., (2018) \\ Luis Antonio Caicedo Hinojosa; Janeth Reina Hurtado Astudillo; Rosario Del Carmen Chuquimarca Chuquimarca; Betthy Narcisa Mazacón Roca}

Teléfono:042384810-042385766

E-mail: ocamposano@lafamilar.com.ec

Delegado por la Universidad:

Nombre: QF. Janeth Hurtado Astudillo Msc.

Cargo: Coordinadora de Prácticas Pre-profesionales en la Facultad de Ciencias de la Salud.

Dirección: Av. Universitaria Km 2 1⁄2 Vía Babahoyo vía Montalvo

Teléfono: 0988794958

Email:_ jhurtado@utb.edu.ec

NOVENA TERMINACIÓN DEL CONVENIO DE PASANTÍA.-

El convenio terminará por una de las siguientes causales:

a. Por cumplimiento del plazo;

b. Por mutuo acuerdo de las partes;

c. Incumplimiento de las obligaciones de la Universidad, establecida con la EMPRESA; y,

d. Por las demás causas previstas en la Ley. 
El desarrollo de competencias profesionales basado en la gestión de medios para la realización de prácticas pre-profesionales en las carreras de la Escuela de Tecnología Médica de la facultad de Ciencias de la Salud

Vol. 2, núm. 1., (2018)

Luis Antonio Caicedo Hinojosa; Janeth Reina Hurtado Astudillo; Rosario Del Carmen Chuquimarca Chuquimarca; Betthy Narcisa Mazacón Roca

\section{DÉCIMA: ACEPTACIÓN.}

Para constancia, libre, voluntariamente y de común acuerdo, las partes declaran expresamente su aceptación a todo lo convenido con el presente convenio, a cuyas estipulaciones se someten, y en tal virtud, lo suscriben en tres ejemplares de idéntico tenor y contenido en Babahoyo, a los 22 días del mes de septiembre 2016.

Dr. Rafael Falconí Montalván, Msc.

RECTOR DE LA

UNIVERSIDAD TÉCNICA DE BABAHOYO
Ing. Carlos Ponce Guzmán.

GERENTE DE LA FAMILIAR

INGENIO ISABEL MARIA. 
El desarrollo de competencias profesionales basado en la gestión de medios para la realización de prácticas pre-profesionales en las carreras de la Escuela de Tecnología Médica de la facultad de Ciencias de la Salud Vol. 2, núm. 1., (2018) Luis Antonio Caicedo Hinojosa; Janeth Reina Hurtado Astudillo; Rosario Del Carmen Chuquimarca Chuquimarca; Betthy Narcisa Mazacón Roca

Formato para la elaboración del informe técnico.

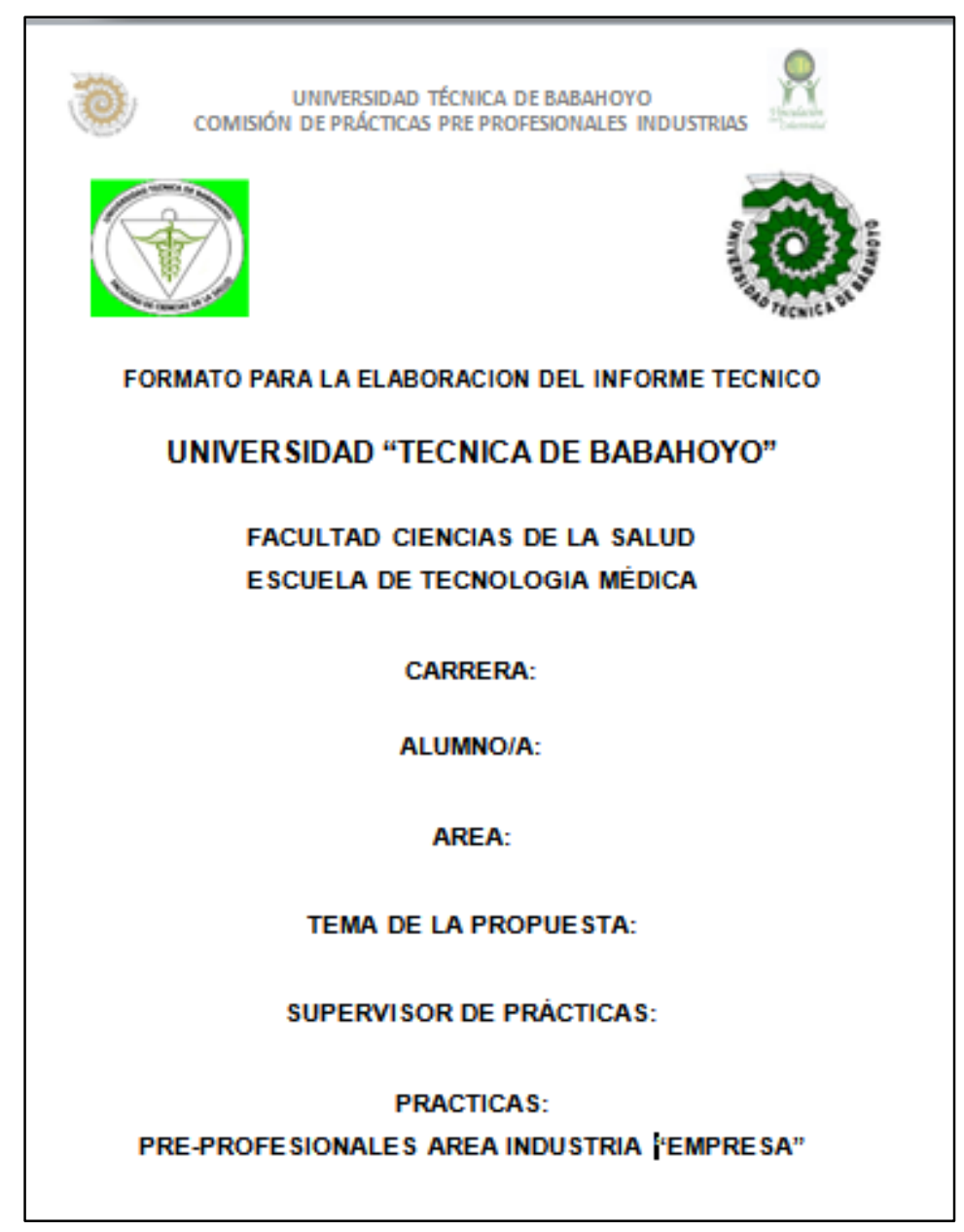

Fuente: Elaboración Propia 
El desarrollo de competencias profesionales basado en la gestión de medios para la realización de prácticas pre-profesionales en las carreras de la Escuela de Tecnología Médica de la facultad de Ciencias de la Salud

Vol. 2, núm. 1., (2018)

Luis Antonio Caicedo Hinojosa; Janeth Reina Hurtado Astudillo; Rosario Del Carmen Chuquimarca Chuquimarca; Betthy Narcisa Mazacón Roca

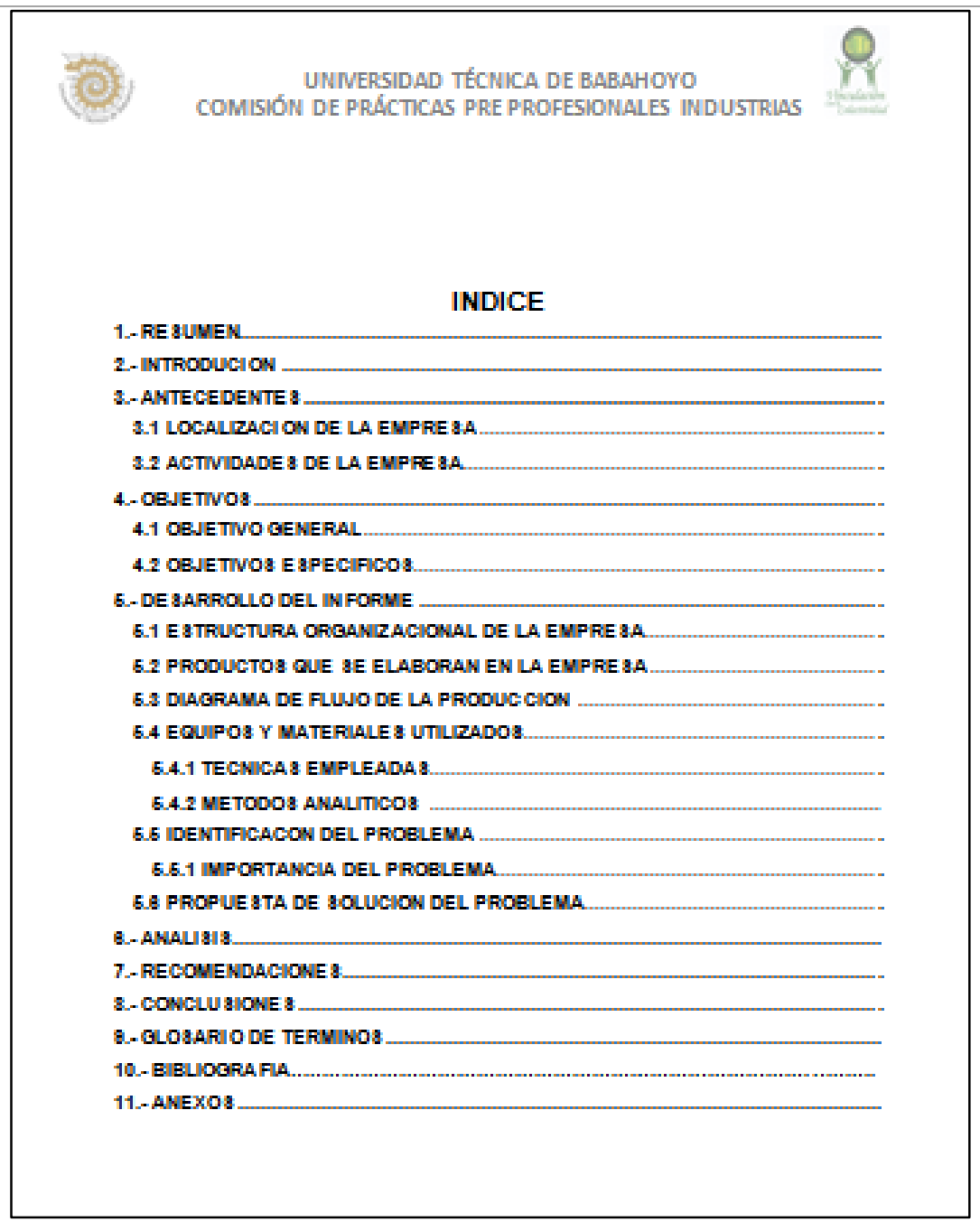

Fuente: Elaboración Propia.

\section{Bibliografía.}

Caicedo, L., Astudillo, J., \& Chuquimarca, R. (2015). Comision de Practicas Pre Profesionales Industrias. Universidad Técnica de Babahoyo. Babahoyo.

Ceroni Galloso , M. (2007). Las prácticas preprofesionales. Revista de la Sociedad Química del Perú, 73(2), 1-2. 


\section{EI desarrollo de competencias profesionales basado en la gestión de medios para la realización de prácticas pre-profesionales en las carreras de la Escuela de Tecnología Médica de la facultad de Ciencias de la Salud}

Vol. 2, núm. 1., (2018)

Luis Antonio Caicedo Hinojosa; Janeth Reina Hurtado Astudillo; Rosario Del Carmen Chuquimarca Chuquimarca; Betthy Narcisa Mazacón Roca

Estévez-Arias, T.-M., Medina-Chicaiza, R.-P., \& González-Hernández, W. (2016). El desarrollo de la motivación profesional en la formación de los estudiantes de periodismo con el uso de las TIC. Revista Iberoamericana de Educación Superior, 12(20), 191-201.

Oliver Ventura, J., Santana Amargó, V., Ferrer Chinea, B., \& Ríos Obregón, J. (2015). LAS PRÁCTICAS PROFESIONALES Y LA FORMACIÓN LABORAL EN LA CARRERA SISTEMA DE INFORMACIÓN EN SALUD. Revista Electrónica "Actualidades Investigativas en Educación", 15(3), 1-18.

Velásquez, W., \& Huatuco, R. (2011). Diagnóstico de las prácticas pre profesionales: caso Facultad de Ingeniería Industrial de la UNMSM. Industrial Data, 14(1), 28-33. 